\title{
Torsional fusional vergences and assessment of cyclodeviation by synoptophore method
}

\author{
D. K. SEN, B. SINGH, AND G. P. MATHUR \\ From the Department of Ophthalmology, Maulana Azad Medical College, Guru Nanak Eye Centre, \\ and New Delhi Tuberculosis Centre, New Delhi, India
}

SUMmary Two special slides, one in front of one eye and one in front of the other eye, were placed in the housings of a synoptophore. The slides were originally designed for the diagnosis and measurement of cyclodeviation, but they were utilised in this study for the measurement of torsional fusional vergence. This was measured by rotating one of the housings of the synoptophore and noting the point of appearance of cyclodiplopia, which was taken as the end point. For measuring vertical torsional fusional vergence the subject was required to fuse the vertical line of the slide with the circle and the vertical line of the slide with the cross, and for measuring horizontal torsional fusional vergence he was required to fuse the horizontal line of the slide with the circle and the horizontal line of the slide with the cross. Incyclovergence was found to be greater than excyclovergence in all the 3 positions of gaze (straight, up, down) studied. The mean amplitude of torsional fusional vergence was lowest in the primary position. Torsional fusional vergence was much less with horizontal lines than with vertical lines in all positions of gaze. The use of the horizontal line of the slide with the cross would be more advantageous for the diagnosis and measurement of cyclodeviation by the synoptophore method, as the amplitude of cyclofusion is minimal in the horizontal meridian.

After the publication of the paper on diagnosis and measurement of cyclodeviation by the synoptophore method with the help of 2 special slides ${ }^{1}$ some ophthalmologists working with these slides experienced difficulties in clinical practice as the upright lines could be fused by some patients. This eliminated some of the torsional error, making maximum cyclodeviation difficult to assess in these patients.

It is granted that torsional fusional vergence may mask some cyclodeviation during its measurement with these slides unless care is taken to reduce it to minimum. One way of doing it is to ask the patient not to make any conscious effort to bring about fusion of the lines but to report as soon as cyclodiplopia is experienced. Moreover, it is accepted that that part of cyclodeviation which can be controlled by normal torsional fusional reserve with ease and comfort needs no treatment and therefore can be ignored in clinical practice. Nevertheless a study was undertaken to find out the amplitude of torsional fusion in normal subjects with vertical and horizontal lines, and that forms the basis of this report.

Correspondence to Dr D. K. Sen, V/4 MAM College Campus, Kotla Road, New Delhi 110002, India.

\section{Subjects and methods}

Forty normal subjects from 18 to 25 years of age with normal binocular functions and without any refractive error were taken for this study.

The subject was placed in front of a synoptophore. Slide 1, a black circle with its circumference marked in degrees and with 2 lines, one vertical and the other horizontal, passing through its centre (Fig. 1), was placed upright before one eye and slide 2, a black cross (Fig. 2), was likewise placed before the other eye. Slides 1 and 2 have been described in detail previously. ${ }^{1}$ The arms of the synoptophore were so set that the small black dot in the intersection of the cross in slide 2 coincided exactly with the small circle in the centre of the large circle in slide 1 , and the vertical and the horizontal lines of the cross in slide 2 coincided exactly with the vertical and the horizontal lines respectively of slide 1 .

For measuring torsional fusion a tilt of the vertical line of the cross in slide 2 was caused by wheel-rotating the slide by means of the torsional deviation screw of the synoptophore. The tilt was gradually increased till a point was reached when the person could no longer maintain fusion of the 2 

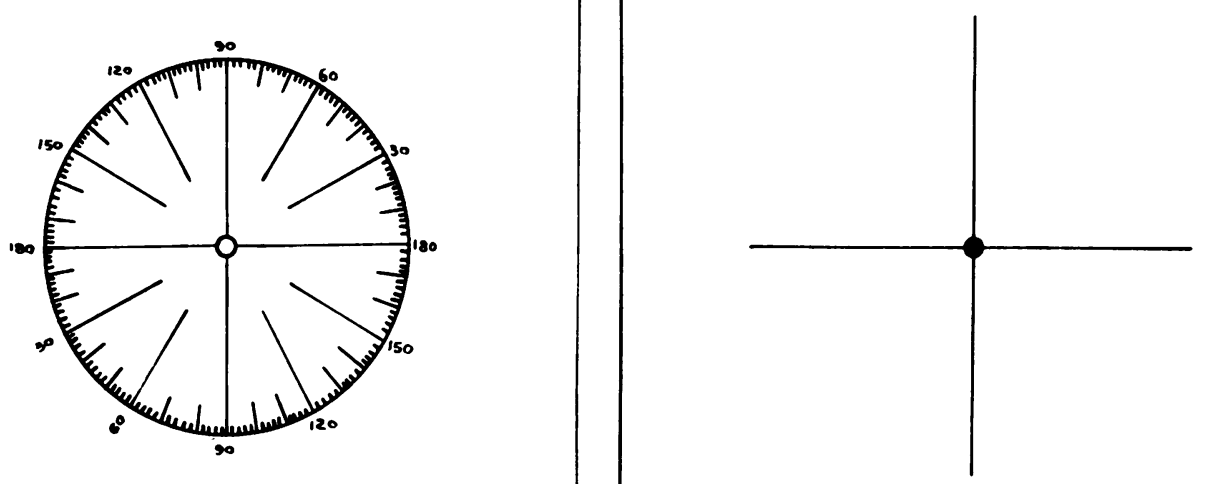

Fig. 1 Slide 1 showing the black circle with the vertical and the horizontal lines

Fig. 2 Slide 2 showing the black cross for use in conjunction with slide 1

In a similar way the torsional fusional vergence vertical lines and reported the appearance of cyclodiplopia. The person was encouraged all the time to maintain fusion of the 2 vertical lines through maximum effort. The amount of tilt of the vertical line of the cross in slide 2 causing cyclodiplopia was read in degrees from a scale provided in the synoptophore. Then a tilt of the vertical line of the cross in slide 2 in the opposite direction was caused by wheel-rotating the slide in the opposite direction, and the whole procedure was repeated with the point when cyclodiplopia appeared taken as the end point. was measured with the horizontal line of the cross in slide $\mathbf{2}$ and the horizontal line in slide 1.

\section{Results}

A set of 12 observations were made on each of 40 subjects covering every combination of 3 positions (primary, looking up, and looking down), 2 axes (vertical and horizontal), and 2 vergences (incyclovergence and excyclovergence). The observed values are summarised in Table 1, which gives

Table 1 Torsional vergences in different positions of gaze in vertical and horizontai meridia

\begin{tabular}{|c|c|c|c|c|c|c|}
\hline & \multicolumn{3}{|c|}{ With vertical lines } & \multicolumn{3}{|c|}{ With horizontal lines } \\
\hline & Min. & Max. & $M e a n \pm S D$ & Min. & $\operatorname{Max}$ & $M e r n \pm S D$ \\
\hline \multicolumn{7}{|l|}{ Primary position: } \\
\hline Incyclovergence & 1 & 15 & $5 \cdot 25 \pm 2 \cdot 73$ & 1 & 6 & $3 \cdot 40 \pm 1 \cdot 28$ \\
\hline Excyclovergence & 1 & 10 & $4 \cdot 15 \pm 1 \cdot 86$ & 1 & 6 & $2 \cdot 58 \pm 1 \cdot 26$ \\
\hline Amplitude & 4 & 20 & $9.40 \pm 3.93$ & 2 & 10 & $5.98 \pm 2.25$ \\
\hline \multicolumn{7}{|l|}{ Looking up by $25^{\circ}$ : } \\
\hline Incyclovergence & 2 & 14 & $5.88 \pm 3.06$ & 1 & 11 & $3.83 \pm 1.97$ \\
\hline Excyclovergence & 1 & 13 & $4.93 \pm 3 \cdot 24$ & 1 & 11 & $2 \cdot 75 \pm 1.75$ \\
\hline Amplitude & 4 & 26 & $10.80 \pm 5.91$ & 2 & 22 & $6.58 \pm 3.55$ \\
\hline \multicolumn{7}{|c|}{ Looking down by $25^{\circ}$ : } \\
\hline Incyclovergence & 1 & 11 & $5.00 \pm 2.57$ & 1 & 8 & $3 \cdot 60 \pm 1.57$ \\
\hline Excyclovergence & 1 & 13 & $4.83 \pm 2.65$ & 1 & 5 & $3 \cdot 18 \pm 1 \cdot 26$ \\
\hline Amplitude & 4 & 23 & $9 \cdot 83 \pm 4.52$ & 2 & 13 & $6 \cdot 78 \pm 2 \cdot 54$ \\
\hline
\end{tabular}


Table 2 Torsional vergences: analysis of variance

\begin{tabular}{|c|c|c|c|c|c|}
\hline Source of variation & $\begin{array}{l}\text { Degrees of } \\
\text { freedom }\end{array}$ & $\begin{array}{l}\text { Sum of } \\
\text { squares }\end{array}$ & $\begin{array}{l}\text { Mean } \\
\text { SS }\end{array}$ & $F$ & $P$ \\
\hline Between positions & 2 & $20 \cdot 33$ & $10 \cdot 16$ & $3 \cdot 56$ & $<0.05$ \\
\hline Between axes & 1 & $381 \cdot 63$ & $381 \cdot 63$ & $133 \cdot 91$ & $\ll 0.001$ \\
\hline Between vergences & 1 & $69 \cdot 00$ & $69 \cdot 00$ & $24 \cdot 21$ & $\ll 0.001$ \\
\hline Between subjects & 39 & $972 \cdot 92$ & $24 \cdot 95$ & $8 \cdot 75$ & $\ll 0.001$ \\
\hline Position/axes interaction & 2 & $7 \cdot 21$ & $3 \cdot 60$ & $1 \cdot 26$ & $>0.05$ \\
\hline Position/vergences interaction & 2 & $12 \cdot 67$ & $6 \cdot 34$ & $2 \cdot 22$ & $>0.05$ \\
\hline Axes/vergences interaction & 1 & 0.04 & 0.04 & 0.01 & $>0.05$ \\
\hline Position/axes/vergences interaction & 2 & 1.49 & $0 \cdot 74$ & $0 \cdot 26$ & $>0.05$ \\
\hline Residual & 429 & $1220 \cdot 64$ & $2 \cdot 85$ & - & - \\
\hline Total & 479 & $2685 \cdot 93$ & - & - & - \\
\hline
\end{tabular}

minimum, maximum, and mean values for each combination as well as similar data for amplitude of vergences.

Further statistical analyses have been carried out by regarding the experiment as one with a factorial design with 3 levels of positions, 2 levels of axes, and 2 vergences. The analysis of variance is set forth in Table 2 .

It can be seen that differences in the 'levels' of all three factors are statistically significant. Values for the primary position are the lowest of the 3 positions studied and are significantly lower than for looking up and looking down positions. Values in the horizontal axes are significantly lower than in the vertical axes. Excyclovergence values are significantly lower than incyclovergence values. None of the interactions is statistically significant.

Taking into account all these findings we can say that the lowest overall value out of the various combinations of positions, axes, and vergences can be had from the primary position, horizontal axes, and excyclovergence. This can also be verified from the actual observations made in this study (Table 1), from which one finds that of all the combinations the lowest mean $(2 \cdot 58)$ was obtained from this particular combination.

A similar analysis was carried out for the amplitude of the vergences for each combination of position and axis. The results are set out in Table 3 .

The differences between the 3 positions fail to attain statistical significance, but there is a highly significant difference between the horizontal and vertical axes. Values for the former are thus found to be lower than for the latter, a difference which was earlier noted in respect of the original values for excyclovergence and incyclovergence in Table 2.

\section{Discussion}

Reported data on torsional fusional vergences are scanty. Though a few studies are available, they are not comprehensive. There is no study in which the torsional fusional vergence has been measured in both vertical and horizontal meridia and in different positions of gaze.

Even in the published data there is some disagreement as to whether incyclovergence ${ }^{2}$ or excyclovergence $^{34}$ is greater. Bullock ${ }^{2}$ found incyclover-

Table 3 Amplitude of torsional vergences: analysis of variance

\begin{tabular}{lccccc}
\hline Source of variation & $\begin{array}{l}\text { Degrees of } \\
\text { freedom }\end{array}$ & $\begin{array}{c}\text { Sum of } \\
\text { squares }\end{array}$ & $\begin{array}{l}\text { Mean } \\
\text { SS }\end{array}$ & \multicolumn{1}{c}{$F$} & $P$ \\
\hline Between positions & 2 & 40.68 & 20.34 & 1.3 & $>0.05$ \\
Between axes & 1 & 763.27 & 763.27 & 49.1 & $\ll 0.001$ \\
Position/axes interaction & 1 & 14.40 & 14.40 & 1.08 & $>0.05$ \\
Residual & 235 & 3653.50 & 15.55 & - & - \\
Total & 239 & 4471.85 & - & - & - \\
\hline
\end{tabular}




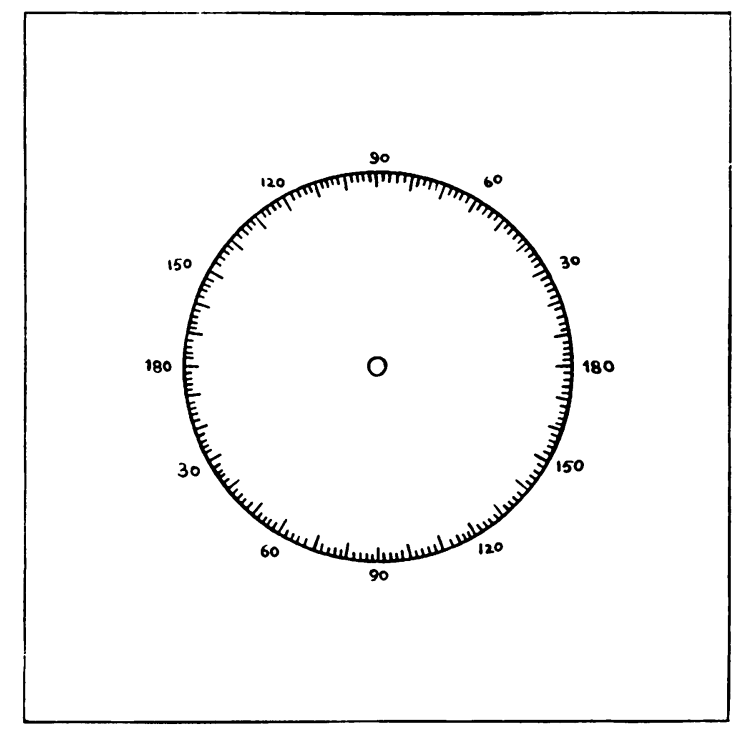

Fig. 3 Modified slide 1 showing that none of the markings on the circumference of the circle extends towards the centre in the form of long lines. This modified slide is now recommended for use in conjunction with slide 2 for the diagnosis and measurement of cyclodeviation

gence to be $6^{\circ}$ to $10^{\circ}$ and excyclovergence to be $4^{\circ}$ to $8^{\circ}$. Parks ${ }^{4}$ stated that a normal person had an incyclovergence of $6^{\circ}$ to $10^{\circ}$ and an excyclovergence of $8^{\circ}$ to $12^{\circ}$. The present study shows that the mean incyclovergence is greater than the mean excyclovergence in all the 3 positions of gaze studied. However, the range of incyclovergence and excyclovergence varied with the positions of gaze and also with the direction of the lines to be fused. In the primary position the range of incyclovergence was between $1^{\circ}$ to $15^{\circ}$ with the vertical lines and between $1^{\circ}$ to $6^{\circ}$ with horizontal lines; the range of excyclovergence was between $1^{\circ}$ to $10^{\circ}$ with the vertical lines and between $1^{\circ}$ and $6^{\circ}$ with horizontal lines.

It has been stated ${ }^{2-5}$ that the amplitude of torsional fusional vergences are between $4^{\circ}$ and $12^{\circ}$. However, our values in the primary position are between $4^{\circ}$ and $20^{\circ}$ with vertical lines and between $2^{\circ}$ and $10^{\circ}$ with horizontal lines. Our study also shows that the amplitude of torsional fusional vergences are much less with horizontally placed lines than with lines vertically placed in all the 3 positions of gaze studied. This finding is interesting and has an important clinical implication in that, while measuring cyclodeviation by the methods previously described $^{1}$ the horizontal line of the cross in slide 2 can be used with greater advantage for bringing out maximum cyclodeviation, as less of it can be masked by torsional fusional vergences. However, it would be still better to modify slide 1 for the purpose of diagnosis and measurement of cyclodeviation so that the markings on the circumference of the circle do not extend towards the centre in the form of long lines. Such extension of the markings has not been found to be of any particular advantage during the testing for cyclodeviation. On the other hand this modification will reduce the impulse for torsional fusion further during the testing for cyclodeviation by our method. The modified slide 1 which is now being used by us for the diagnosis and measurement of cyclodeviation by synoptophore method is shown in Fig. 3.

\section{References}

${ }^{1}$ Sen DK, Singh B, Shroff NM. Diagnosis and measurement of cyclodeviation. Br J Ophthalmol 1977; 61: 690-2.

${ }^{2}$ Bullock K, Bredemeyer HG. Orthoptics: Theory and Practice. St Louis: Mosby, 1968: 75.

${ }^{3}$ Kramer ME. Clinical Orthoptics, Diagnosis and Treatment, 2nd ed. St Louis: Mosby, 1953: 480.

${ }^{4}$ Parks MM. Ocular Motility and Strabismus. New York: Harper Row, 1975: 65.

${ }^{5}$ Lyle TK. Torsional diplopia due to cyclotropia and its surgical Treatment. Trans Am Acad Ophthalmol Otolaryngol $1964 ; 68: 387-411$. 